\title{
Shape optimization analysis considering a rotational body in a flow field based on the adjoint variable and the finite element methods
}

\author{
Takahiko KURAHASHI*, Yuta OZEKI* and Eiji KATAMINE** \\ * Department of Mechanical Engineering, Nagaoka University of Technology \\ 1603-1 Kamitomiokamachi, Nagaoka-shi, Niigata, 940-2137, Japan \\ E-mail:kurahashi@mech.nagaokaut.ac.jp \\ ** Department of Mechanical Engineering, National Institute of Gifu College \\ 2236-2 Kamimakuwa, Motosu-shi, Gifu, 501-0495, Japan
}

Received: 4 December 2018; Revised: 27 January 2019; Accepted: 26 March 2019

\begin{abstract}
In this study, we present a shape optimization analysis considering a rotational body in a flow field based on the adjoint variable and the finite element methods. The purpose of this study is to obtain an optimal shape so as to closely approach the target velocity in the target regions. It is necessary to apply the correct boundary conditions to the boundaries of the rotational structure to be able to obtain an appropriate shape that satisfies the conditions of the target velocity. It is also important to determine the position of the target domain given the target velocity. Based on these considerations, we have constructed a program code for shape optimization analysis considering rotational body in a flow field. Freefem ++ was used to calculate the optimal shape.
\end{abstract}

Keywords: Shape optimization, Adjoint variable method, Finite element method, Incompressible viscous fluid field, Rotational body

\section{Introduction}

In this study, an investigation is carried out into the shape optimization problem of the outer shape of a flow channel that includes a rotational body. The shape optimization problem in Stokes flow is theoretically explained: it is known that the optimal shape is that of a rugby ball for the drag minimization problem in Stokes flow (Pironneau, 1973, 1974, 1984). When implementing the Navier-Stokes equations using finite element analysis, it is necessary to satisfy the Ladyzhenskaya-Babuska-Brezzi condition (hereinafter 'LBB condition'). The mixed interpolation method and the stabilized finite element method are generally employed when considering the LBB condition. Shape optimization analysis of incompressible viscous flow is increasingly being carried out using the stabilized bubble function FEM (Matsumoto, 2003). Even if this technique is used in shape optimization analysis, it is known that the sensitivity distribution with respect to the coordinate of the target body is oscillated on the boundary of the target body. The shape optimization analysis of a body located in an unsteady-state viscous flow field was also investigated by Yagi and Kawahara using the stabilized bubble function FEM (Yagi and Kawahara, 2005, 2007). The smoothing technique in which the sensitivity, i.e., the gradient of the Lagrange function with respect to the coordinates on the design boundary, is modified to the second order of differentiable sensitivity (Jameson, 2003) is employed in this study. Their study was extended to shape optimization analysis in a compressible flow field (Nakajima and Kawahara, 2008, 2010a, 2010b). However, the traction method is also applied to control the sensitivity oscillation (Azegami, 1994, 2014). The traction method is applied to numerous shape optimization problems, such as the channel shape optimization problem considering minimization of dissipation energy (Katamine et al., 1994, 1995a, 2005); that in potential flow (Katamine et al., 1995b, 1998); in the heat conduction field (Katamine et al., 1999, 2000, 2008a, 2010); for thermoelastic solids (Katamine et al., 2006, 2011, 2017); in the heat convection field (Katamine et al., 2007, 2013, 2016); for that of a body located in a steady-state viscous flow field considering drag minimization and lift maximization (Katamine et al., 2008b) and fir the stability of a Navier-Stokes flow 
field (Kiriyama et al., 2018) . It has been confirmed that smoothing of the sensitivity distribution can be appropriately carried out and the optimal shape can be obtained numerically.

Based on this knowledge, we extend here the shape optimization problem in an unsteady-state viscous flow field to that considering a rotational body. Numerous everyday mechanical devices include rotational bodies, e.g., turbojet engines, air conditioners, and heat sinks. However, it is difficult to find technical papers on the shape optimization problem for these mechanical devices. We therefore anticipate that the results of this study will contribute to the design of mechanical devices of this type. The share-slip mesh update method is frequently employed in flow simulations that include a rotational body (Behr and Tezduyar, 1999). In the present study, the shear-slip mesh update method is employed for the analysis of unsteady-state viscous flow fields considering a rotational body. The mixed interpolation method is used in the flow analysis, and the traction method is employed in the shape optimization problem. The Freefem ++ program code is used in this study (Hecht, 2012, Ootsuka, 2014).

\section{Formulations for the shape optimization problem}

\subsection{Derivation of the stationary condition of the Lagrange function}

The performance function is first defined to obtain the shape that will optimally satisfy the objectives. In this study, it is defined as shown in Eq. (1) considering the objective of the flow velocity being close to the target velocity in the target regions.

$$
J=\frac{1}{2} \int_{t_{0}}^{t_{f}} \int_{\Omega} Q_{i}\left(u_{i}-u_{i(\text { target })}\right)^{2} d \Omega d t
$$

where $Q_{i}, u_{i}$ and $u_{i(\text { target })}$ indicate weight constant, computed flow velocity and target flow velocity. $t_{0}, t_{f}$ and $\Omega$ denote initial time, terminal time and computational domain. The weight constant $Q_{i}$ is given as 1 in the target regions, and as 0 in other regions. The target flow velocity $u_{i(t a r g e t)}$ is a preassigned condition in shape optimization analysis, so it is necessary to give this condition appropriately to be able to obtain the optimized shape: this is because the target flow velocity $u_{i(t a r g e t)}$ is affected by the inflow and the outflow boundary conditions, the Reynolds number and the angular velocity of the body. The incompressible Navier-Stokes equation shown in Eqs. (2) and (3) are employed as the governing equations.

$$
\begin{aligned}
& \dot{u}_{i}+u_{j} u_{i, j}+p_{, i}-\frac{1}{R e} u_{i, j j}=0 \quad \text { in } t \in\left[t_{0}, t_{f}\right] \text { in } \Omega \\
& u_{i, i}=0 \quad \text { in } \quad t \in\left[t_{0}, t_{f}\right] \quad \text { in } \Omega
\end{aligned}
$$

The initial and boundary conditions shown in Eqs. (4), (5) and (6) are introduced as constraint conditions for the performance function. The area constraint condition shown in Eq. (7) is also considered in this study.

$$
\begin{aligned}
& u_{i}=\hat{u}_{i} \quad \text { at } t=t_{0} \quad \text { in } \Omega \\
& u_{i}=\hat{u}_{i} \quad \text { in } \quad t \in\left[t_{0}, t_{f}\right] \text { on } \Gamma_{1}, \Gamma_{\text {rotation }} \text { and } \Gamma_{\text {design }} \\
& t_{i}=\hat{t}_{i}=\left\{-p \delta_{i j}+\frac{1}{R e} u_{i, j}\right\} n_{j} \text { in } t \in\left[t_{0}, t_{f}\right] \text { on } \Gamma_{2} \\
& \sum_{i=1}^{m x} A_{i}=A_{\text {initial }} \quad \text { in } \Omega
\end{aligned}
$$

where $u_{i}, p, R e, \delta_{i j}$ and $n_{i}$ indicate flow velocity, pressure, Reynolds number, Dirac's delta function and direction cosine of the unit outward normal of the boundary, respectively. $\Gamma_{1}, \Gamma_{2}, \Gamma_{\text {rotation }}$ and $\Gamma_{\text {design }}$ represent the Dirichlet, the Neumann, the rotational body and the design boundaries. On the rotational boundary, the flow velocity is given as $u_{1}=-\omega r \sin \theta$, $u_{2}=\omega r \cos \theta$. The parameters $\omega, r$ and $\theta$ indicate the angular velocity, distance from rotational center point and the angle for the rotational center point, respectively. In addition, in Eq. (7), $m x$ and $A_{\text {initial }}$ represent the total number of elements and the area of the whole domain except for the rotational body at initial iteration. The boundary condition applied to $\Gamma_{\text {rotation }}$ is referred to as the adhesive boundary condition (Imai, 1973). Introducing the adjoint variables, the performance function is extended to Eq. (8), which is referred to as the Lagrange function.

$$
J^{*}=J+\int_{t_{0}}^{t_{f}} \int_{\Omega} u_{i}^{*}\left\{\dot{u}_{i}+u_{j} u_{i, j}+p_{, i}-\frac{1}{R e} u_{i, j j}\right\} d \Omega d t+\int_{t_{0}}^{t_{f}} \int_{\Omega} p^{*} u_{i, i} d \Omega d t=\int_{t_{0}}^{t_{f}} L d t
$$


where $u_{i}^{*}$ and $p_{i}^{*}$ respectively indicate the adjoint variables for the flow velocity and pressure. The first variation of the Lagrange function must be zero to satisfy the stationary condition, and is written as Eq. (9).

$$
\begin{aligned}
\delta J^{*} & =\int_{t_{0}}^{t_{f}} \int_{\Omega} \delta u_{i}^{*}\left\{\dot{u}_{i}+u_{j} u_{i, j}+p_{, i}-\frac{1}{R e} u_{i, j j}\right\} d \Omega d t+\int_{t_{0}}^{t_{f}} \int_{\Omega} \delta p^{*} u_{i, i} d \Omega d t \\
& +\int_{t_{0}}^{t_{f}} \int_{\Omega} \delta u_{i}\left\{-\dot{u}_{i}^{*}+u_{j, i} u_{j}^{*}-\left(u_{j} u_{j}^{*}\right)_{, j}-p_{, i}^{*}-\frac{1}{R e} u_{i, j j}^{*}+Q\left(u_{i}-u_{i(\text { target })}\right)\right\} d \Omega d t+\int_{t_{0}}^{t_{f}} \int_{\Omega} \delta p\left(-u_{i, i}^{*}\right) d \Omega d t \\
& +\int_{t_{0}}^{t_{f}} \int_{\Gamma} \delta u_{i}\left\{u_{j} u_{i}^{*}+p^{*} \delta_{i j}+\frac{1}{R e} u_{i, j}^{*}\right\} n_{j} d \Gamma d t \\
& +\int_{t_{0}}^{t_{f}} \int_{\Gamma} \delta u_{i}^{*}\left\{p \delta_{i j}-\frac{1}{R e} u_{i, j}\right\} n_{j} d \Gamma d t \\
& +\int_{\Omega} \delta u_{i}\left(t_{f}\right) u_{i}^{*}\left(t_{f}\right) d \Omega-\int_{\Omega} \delta u_{i}\left(t_{0}\right) u_{i}^{*}\left(t_{0}\right) d \Omega=0
\end{aligned}
$$

Considering the stationary condition of the Lagrange function, the adjoint equation and the conditions for adjoint variables shown in Eqs. (10) - (14) are obtained.

$$
\begin{aligned}
& -u^{*}{ }_{i}+u_{j, i} u_{j}^{*}-\left(u_{j} u_{i}^{*}\right)_{, j}-p_{, i}^{*}-\frac{1}{R e} u_{i, j j}^{*}+Q_{i}\left(u_{i}-u_{i(\text { target })}\right)=0 \text { in } t \in\left[t_{0}, t_{f}\right] \text { in } \Omega \\
& u^{*}{ }_{i, i}=0 \text { in } t \in\left[t_{0}, t_{f}\right] \quad \text { in } \Omega \\
& u_{i}^{*}=0 \text { at } t=t_{f} \quad \text { in } \Omega \\
& u_{i}^{*}=0 \text { in } t \in\left[t_{0}, t_{f}\right] \quad \text { on } \Gamma_{1}, \Gamma_{\text {rotation }} \text { and } \Gamma_{\text {design }} \\
& t_{i}^{*}=t_{i}^{*}=\left\{u_{j} u_{i}^{*}+p^{*} \delta_{i j}+\frac{1}{R e} u_{i, j}^{*}\right\} n_{j} \text { in } t \in\left[t_{0}, t_{f}\right] \text { on } \Gamma_{2}
\end{aligned}
$$

The gradient of the Lagrange function with respect to the coordinates on the design boundary, $G_{i}$, is derived by Eq. (15).

$$
\begin{aligned}
\int_{t_{0}}^{t_{f}} \int_{\Gamma} t_{i}^{*} \delta u_{i} d \Gamma d t & =\int_{t_{0}}^{t_{f}} \int_{\Gamma_{1}+\Gamma_{2}+\Gamma_{\text {rotation }}} t_{i}^{*} \delta u_{i} d \Gamma d t+\int_{t_{0}}^{t_{f}} \int_{\Gamma_{\text {design }}} t_{i}^{*} u_{i, j} \delta x_{j} d \Gamma d t \\
& =\int_{t_{0}}^{t_{f}} \int_{\Gamma_{1}+\Gamma_{2}+\Gamma_{\text {rotation }}} t_{i}^{*} \delta u_{i} d \Gamma d t+\int_{t_{0}}^{t_{f}} \int_{\Gamma_{\text {design }}}\left\{u_{j} u_{i}^{*}+p^{*} \delta_{i j}+\frac{1}{R e} u_{i, j}^{*}\right\} n_{j} u_{i, j} \delta x_{j} d \Gamma d t \\
& =\int_{t_{0}}^{t_{f}} \int_{\Gamma_{1}+\Gamma_{2}+\Gamma_{\text {rotation }}} t_{i}^{*} \delta u_{i} d \Gamma d t+\int_{\Gamma_{\text {design }}} G_{j} \delta x_{j} d \Gamma
\end{aligned}
$$

The gradient $G_{i}$ is modified by the traction method, in which the gradient $G_{i}$ is employed as the external force for the linear elastic body, and the displacement value is used as the modified gradient $G_{i}^{*}$. The governing equation for the deformation of the linear elastic body is shown in Eqs. (16), (17) and (18), and the boundary conditions are shown in Eqs. (19) and (20).

$$
\begin{aligned}
& \sigma_{i j, j}=0 \text { in } \Omega \\
& \varepsilon_{i j}=\frac{1}{2}\left(G_{i, j}^{*}+G_{j, i}^{*}\right) \text { in } \Omega \\
& \sigma_{i j}=D_{i j k l} \varepsilon_{k l} \text { in } \Omega \\
& G_{i}^{*}=0 \text { on } \Gamma_{1}, \quad \Gamma_{2} \text { and } \Gamma_{\text {rotation }} \\
& t_{i}=G_{i} \text { on } \Gamma_{\text {design }}
\end{aligned}
$$

where $\sigma_{i j}, \varepsilon_{i j}$ and $D_{i j k l}$ indicate the stress component, the strain component and the elastic matrix. The non-dimensional Young's modulus $E$ and the Poisson ratio $v$ in the elastic matrix are given as $E=1$ and $v=0$, respectively. The elastic matrix is written as Eq. (21).

$$
[D]=\left[\begin{array}{lll}
1 & 0 & 0 \\
0 & 1 & 0 \\
0 & 0 & 1
\end{array}\right]
$$

Finally, the coordinates on the target boundary are updated by using the modified gradient $G_{i}^{*}$ as shown in Eq. (22).

$$
x_{i}^{(l+1)}=x_{i}^{(l)}-\eta G_{i}^{*(l)} \text { in } \Omega
$$

where $\eta$ indicates the step length. 


\subsection{Derivation of the weak form for the governing and the adjoint equations}

The weighted residual equation for the governing equation is written as Eqs. (23) and (24).

$$
\begin{aligned}
& \int_{\Omega_{e}} W_{u_{i}}\left\{\dot{u}_{i}+u_{j} u_{i, j}+p_{, i}-\frac{1}{R e} u_{i, j j}\right\} d \Omega=0 \\
& \int_{\Omega_{e}} W_{p} u_{i, i} d \Omega=0
\end{aligned}
$$

where $W_{u_{i}}, W_{p}$ and $\Omega_{e}$ indicate the weighting function for the flow velocity, the pressure and the element domain. Similarly, the weighted residual equation for the adjoint equation is written as Eqs. (25) and (26).

$$
\begin{aligned}
& \int_{\Omega_{e}} W_{u_{i}}^{*}\left\{-\dot{u}^{*}{ }_{i}+u_{j, i} u_{j}^{*}-\left(u_{j} u_{i}^{*}\right)_{, j}-p_{, i}^{*}-\frac{1}{R e} u_{i, j j}^{*}+Q_{i}\left(u_{i}-u_{i(\text { target })}\right)\right\} d \Omega=0 \\
& \int_{\Omega_{e}} W_{p}^{*} u^{*}{ }_{i, i} d \Omega=0
\end{aligned}
$$

where $W_{u_{i}}^{*}$ and $W_{p}^{*}$ indicate the weighting function for the adjoint variables. Applying the Green theorem to Eq. (23), the weak form of the weighted residual equation is obtained via Eq. (27).

$$
\int_{\Omega_{e}}\left\{W_{u_{i}} \dot{u}_{i}+W_{u_{i}} u_{j} u_{i, j}-W_{u_{i}, j} p+\frac{1}{R e} W_{u_{i}, j} u_{i, j}\right\} d \Omega=\int_{\Gamma_{e}} W_{u_{i}} t_{i} d \Gamma
$$

Similarly, the weak form for Eq. (25) is obtained via Eq. (28).

$$
\int_{\Omega_{e}}\left\{-W_{u_{i}}^{*} \dot{u}_{i}^{*}+W_{u_{i}}^{*} u_{j, i} u_{j}^{*}+W_{u_{i}, j}^{*} u_{j} u_{i}^{*}+W_{u_{i}, i}^{*} p^{*}+\frac{1}{R e} W_{u_{i}, j}^{*} u_{i, j}^{*}+W_{u_{i}}^{*} Q_{i}\left(u_{i}-u_{i(\operatorname{target})}\right)\right\} d \Omega=\int_{\Gamma_{e}} W_{u_{i}}^{*} t_{i}^{*} d \Gamma
$$

The backward difference method is applied to Eqs. (27) and (28). For the interpolation of the state and the adjoint variables, the first order triangular element is employed for the variables $p, W_{p}, p^{*}$ and $W_{p}^{*}$, and the second order triangular element is used for the variables $u_{i}, W_{u_{i}}, u_{i}^{*}$ and $W_{u_{i}}^{*}$ (See Fig. 1.).
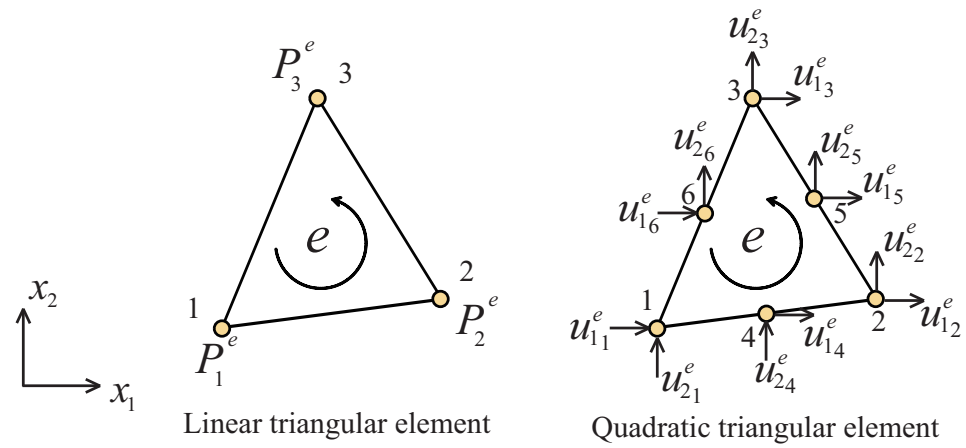

Fig. 1 Element types for pressure and velocity. In this study, the mixed interpolation method is applied to discretize the governing equation, and the linear triangular and the quadratic triangular elements are employed for the pressure and velocity, respectively.

\subsection{Computational flow}

The computational flow for shape optimization is shown below.

(1) Select the initial surface coordinates $x_{i}$ on design boundary $\Gamma_{\text {design }}$ and the convergence criterion $\varepsilon$ and number of iterations $(l)=0$.

(2) Solve the Navier-Stokes equation, the equation superposed by Eqs. (27) and (24) under the initial and the boundary conditions ,i.e., Eqs. (4), (5) and (6).

(3) Compute the performance function, i.e., Eq. (1). If the value of the judgment equation $\left|\frac{J^{(l+1)}-J^{(l)}}{J^{(0)}}\right|$ is lower than convergence criterion $\varepsilon$, this computation finalizes. Otherwise, go to step (4).

(4) Solve the adjoint equation, the equation superposed by Eqs. (28) and (26) under the terminal and the boundary conditions ,i.e., Eqs. (12), (13) and (14).

( 5 ) Compute the gradient of the Lagrange function with respect to the surface coordinates on the design boundary Eq. (15).

(6) Compute the modified gradient based on the traction method,i.e., Eqs. (16) - (20). 
( 7 ) Update the surface coordinates on the design boundary using the modified gradient ,Eq. (22) and return to step (2).

In step (7), the surface coordinates on the design boundary $\Gamma_{\text {design }}$ are updated to satisfy the area constraint condition shown in Eq.(7).

\section{Numerical experiments on shape optimization considering a rotational body}

\subsection{Considerations for adhesive boundary condition}

Examination by the adhesive boundary condition is performed using the computational model shown in the References (Chen et al., 1991). The computational conditions are shown in Table 1, and the computational model and the boundary conditions are shown in Fig. 2. The finite element mesh is shown in Fig. 3. In the numerical test, the parameter $\alpha$ is calculated as $\alpha=\frac{\omega r}{U}$, the radius of the cylinder $r$ is given as 1.0, the inflow velocity $U$, i.e., the boundary condition, is given as 1.0, and the non-dimensional angular velocity $\omega$ is adjusted to $0.5,1.0$ and 2.07.

As the computational result, Fig. 4 show the velocity and the pressure distribution at $T=24.0$. The vector indicates the flow velocity, and the contours denote the pressure value. It can be seen that the velocity and the pressure distribution are appropriately obtained. Figure 5 show a comparison of the time history of the drag and lift coefficients. The drag and lift coefficients are calculated according to the drag and lift forces, and the method shown in a paper by Tabata (1998) is used to calculate the drag and lift forces. It can be seen that the results arrived at using the present method are in good agreement with the results shown in the References (Chen et al., 1991). If it is also necessary to obtain a more accurate match between the present method and that of Chen et al., the adaptive remeshing technique should be employed.

Table 1 Computational conditions. In each non-dimensional angular velocity $\omega$, same computational conditions are employed.

\begin{tabular}{cc}
\hline Non-dimensional time increment $\Delta t$ & 0.01 \\
Time steps & 2400 \\
Number of nodes & 11496 \\
Number of elements & 22692 \\
Reynolds number & 200 \\
\hline
\end{tabular}

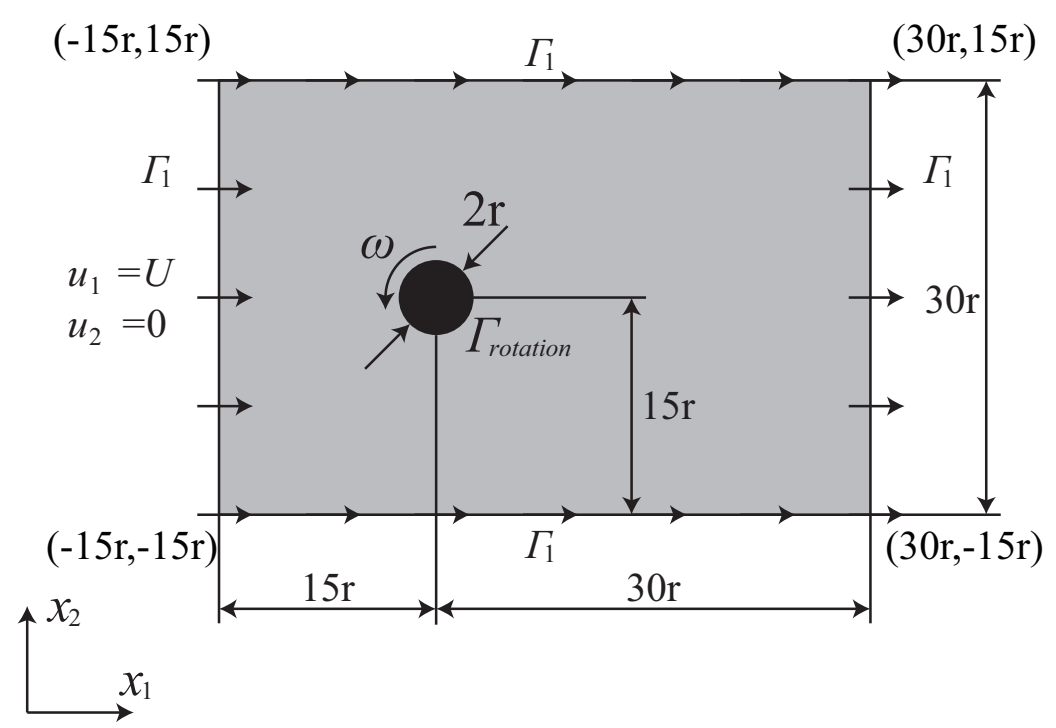

Fig. 2 Computational model and boundary conditions. The flow velocity is given from the boundary on the lefthand side. The non-dimensional angular velocity $\omega$ is adjusted in numerical experiments, the flow field is compared in each case. 


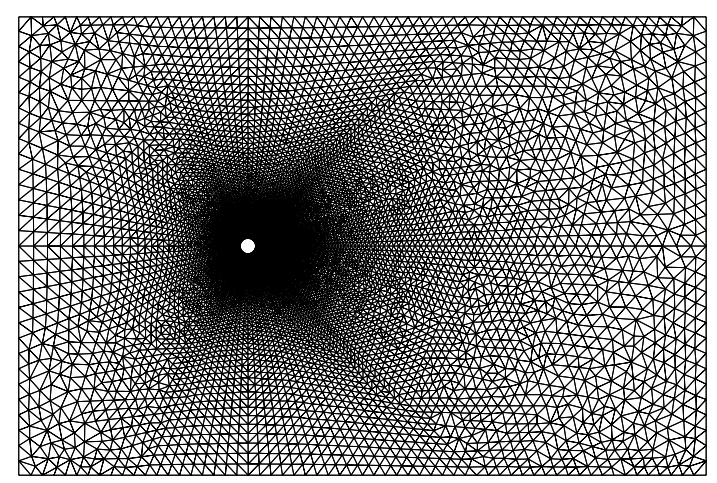

(a) Whole domain.

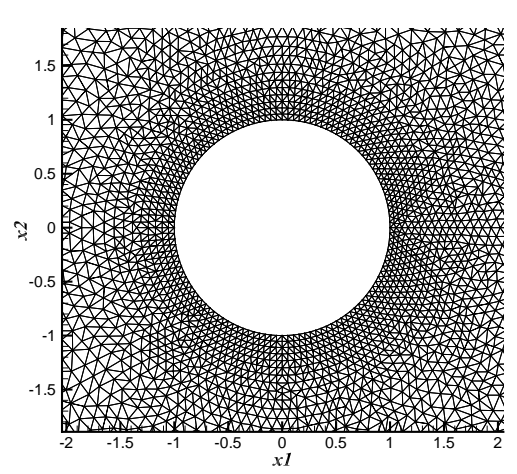

(b) Enlarged version near the cylinder

Fig. 3 Finite element mesh. The computational domain is subdivided by unstructured triangular meshes, and the fine mesh is applied to compute the flow field high accurately around rotational cylinder.

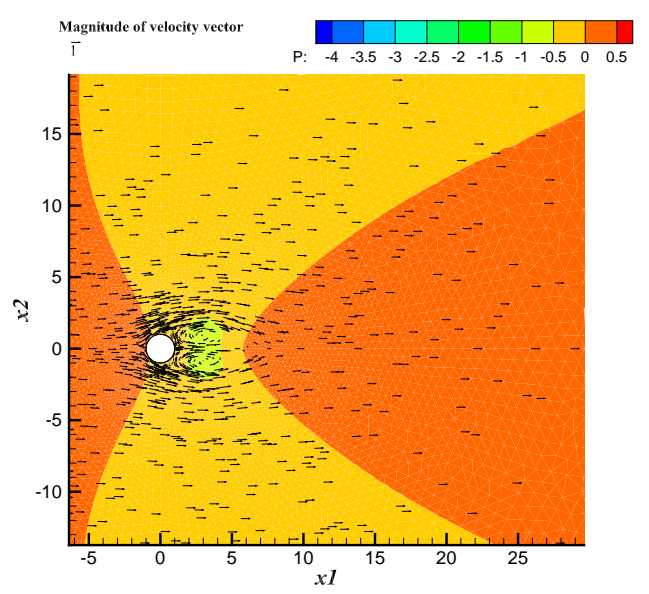

(a) In case of no rotation.

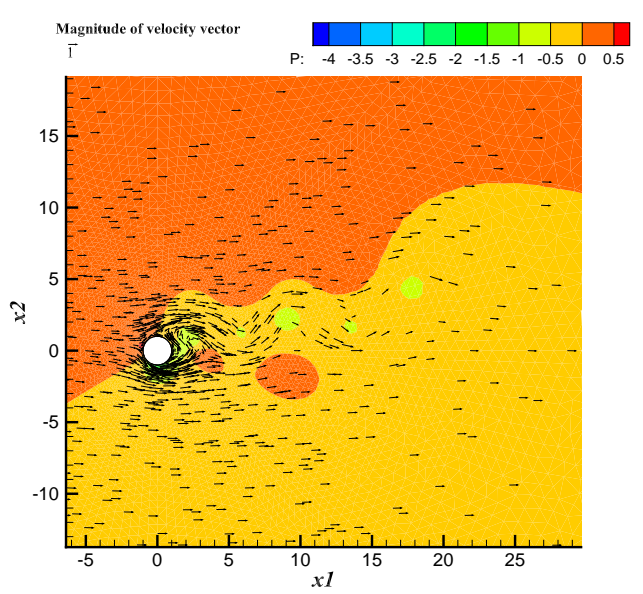

(c) In case of $\alpha=1.0$.

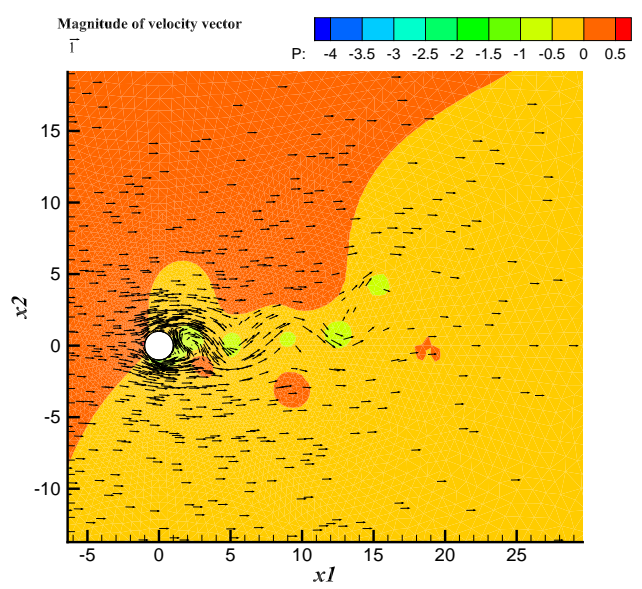

(b) In case of $\alpha=0.5$.

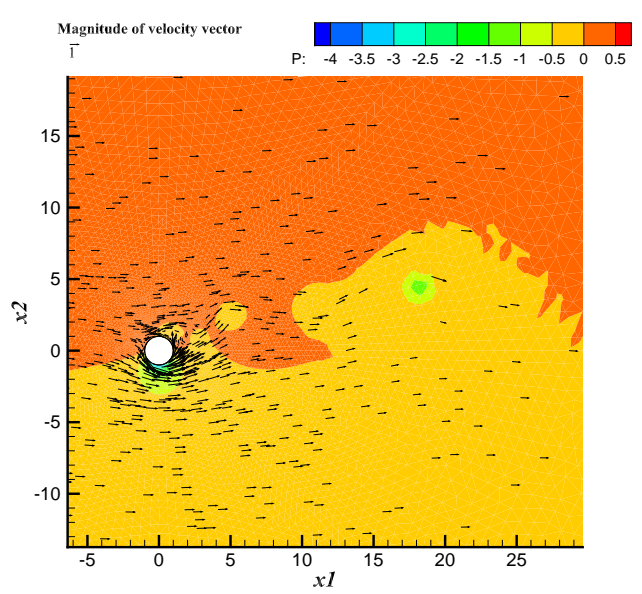

(d) In case of $\alpha=2.07$.

Fig. 4 Velocity and pressure distribution at $T=24.0$. From this result, it is seen that the distribution of velocity vector and pressure contour is difference in case of each non-dimensional angular velocity $\omega$. 


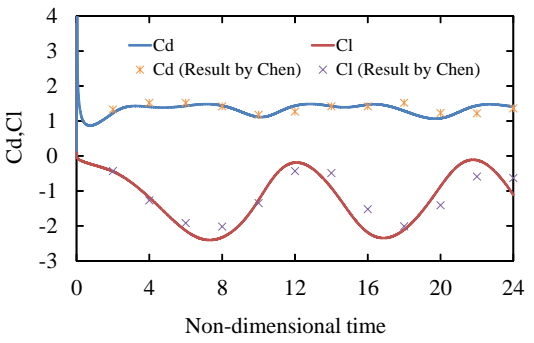

(a) In case of $\alpha=0.5$.

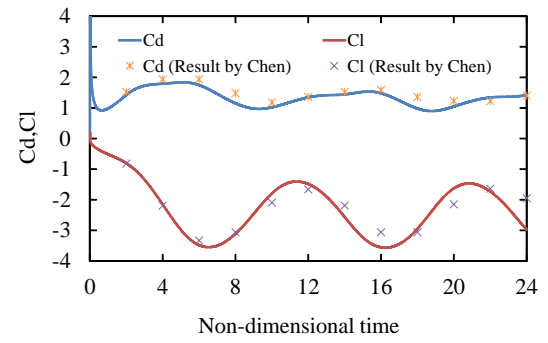

(b) In case of $\alpha=1.0$.

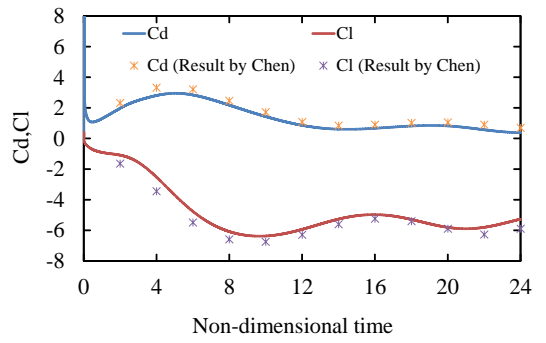

(c) In case of $\alpha=2.07$.

Fig. 5 Comparison of time history of drag and lift coefficients. Plots and lines are results by Chen et al. and present method, respectively. It is seen that the tendency of result by present method is in good agreement with the result by Chen et al..

\subsection{Numerical tests for shape identification considering a rotational body}

Shape identification analysis considering a rotational body is carried out. The computational conditions are shown in Table 2, and the computational model and the boundary conditions are shown in Fig. 6. The non-dimensional angular velocities $\omega$ are given as $2 \pi, 6 \pi$ and $10 \pi$. The finite element mesh, i.e., the initial mesh in the shape identification analysis, is shown in Fig. 7. When $\omega=10 \pi$, the fine mesh is used in comparison with the case of $\omega=2 \pi$ and $\omega=6 \pi$ to prevent divergence of the flow analysis. The target shape in the shape identification analysis is shown in Fig. 8, and the computed flow velocities in the target regions are used in the shape identification analysis. In the target regions, the weighting constant $Q_{i}$ is given as 1.0 in the target regions; otherwise the weighting constant $Q_{i}$ is given as 0.0 . In addition, the nodes in target regions are not updated in each iteration, and other nodes are updated based on the traction method.

As the computational result, Fig. 9 shows the variation in both the performance function and area of the target domain. It can be seen that the value of the performance function gradually decreases and converges for all angular velocities, with the area of the target domain remaining constant. A comparison of the initial shape and identified shape is shown in Fig. 10. It can be seen that the initial shape approaches the target shape in all cases, and the identified shape is independent of the non-dimensional angular velocity $\omega$ given as $2 \pi, 6 \pi$ and $10 \pi$. Figure 11 shows the time history of the velocity at the center point in target regions A, B and C, respectively. For all angular velocities, it is found that the result for the identified shape approaches the result for the target shape more closely than does the result for the initial shape.

Table 2 Computational conditions. In each non-dimensional angular velocity $\omega$, same computational conditions are employed excepting for the finite element mesh.

\begin{tabular}{ccc}
\hline & $\omega=2 \pi, 6 \pi$ & $\omega=10 \pi$ \\
\hline Non-dimensional time increment $\Delta t$ & 0.01 & 0.01 \\
Time steps & 800 & 800 \\
Number of nodes & 11552 & 25358 \\
Number of elements & 22578 & 49928 \\
Reynolds number $R e$ & 200 & 200 \\
Convergence criterion $\epsilon$ & 0.001 & 0.001 \\
Step length $\eta$ & 0.01 & 0.01 \\
\hline
\end{tabular}




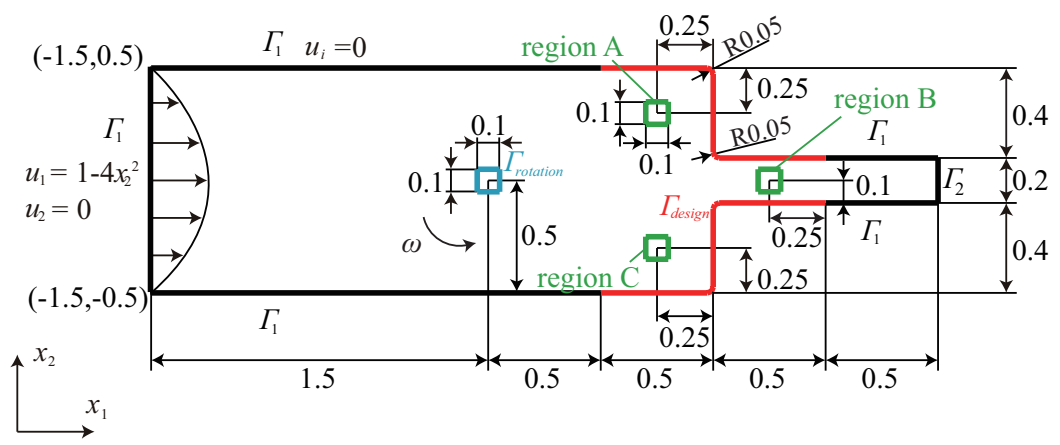

Fig. 6 Computational model, target regions and boundary condition. The flow velocity is given from the boundary on the left-hand side. The position of rotational body and the target regions are shown in this figure.

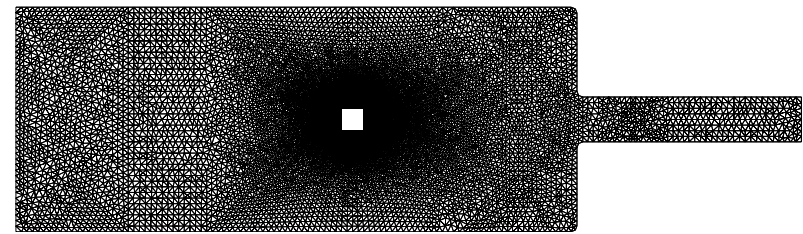

(a) In case of $\omega=2 \pi$ and $\omega=6 \pi$.

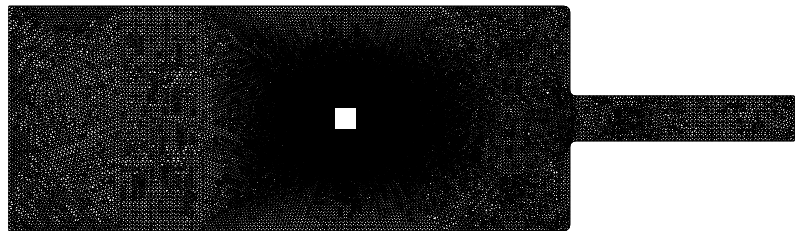

(b) In case of $\omega=10 \pi$.

Fig. 7 Finite element mesh. In case of $\omega=10 \pi$, fine mesh is employed in comparison with the cases of $\omega=2 \pi$ and $\omega=6 \pi$.

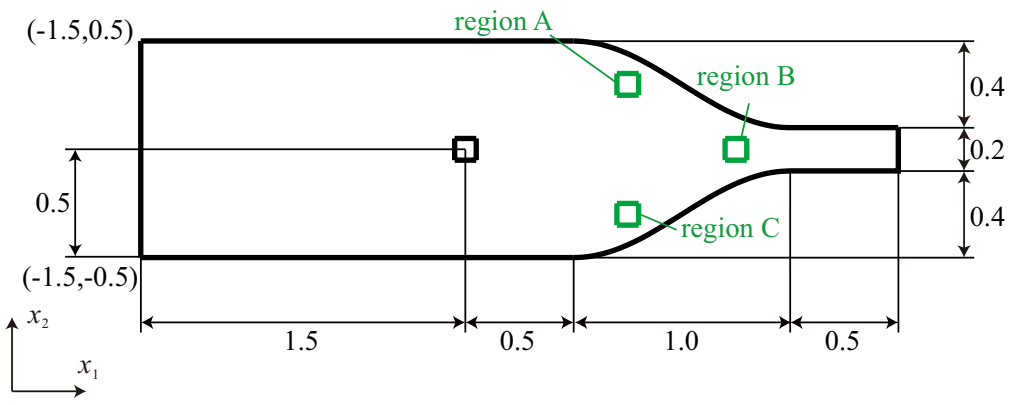

Fig. 8 Target shape. The flow velocity in this model is given as the target velocity. In the shape optimization, the iterative computation is carried out such that the non-dimensional velocity is agreement with the target velocity.

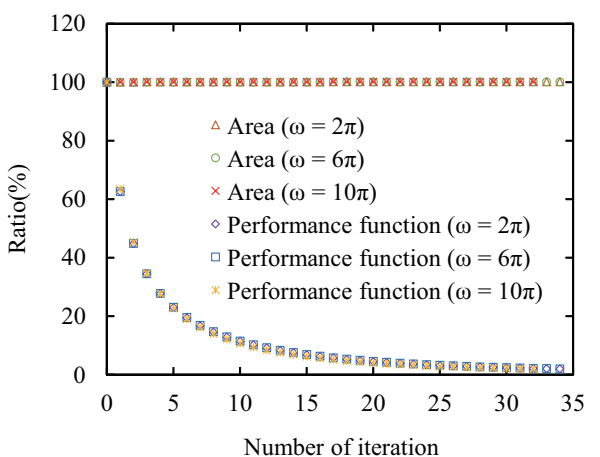

Fig. 9 Variation of both performance function and area for $\omega=2 \pi, 6 \pi$ and $10 \pi$. In each case of $\omega$ it is found that the area constraint condition is satisfied, and the performance function gradually decreases and finally converges.

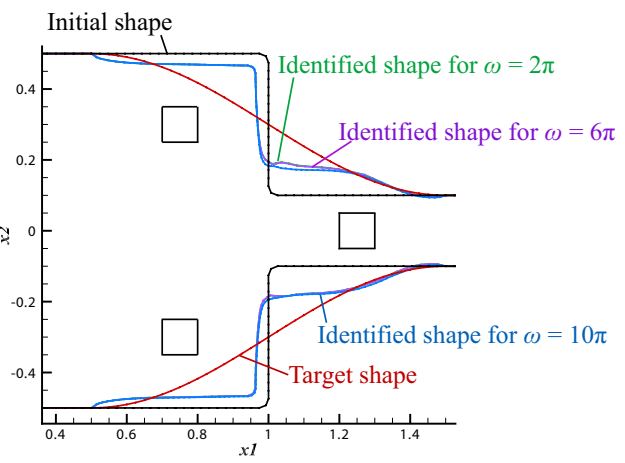

Fig. 10 Comparison of initial shape, identified shape and target shape for $\omega=2 \pi, 6 \pi$ and $10 \pi$.

From this result, it is found that the identified shape is approached to the target shape in comparison with the initial shape. 


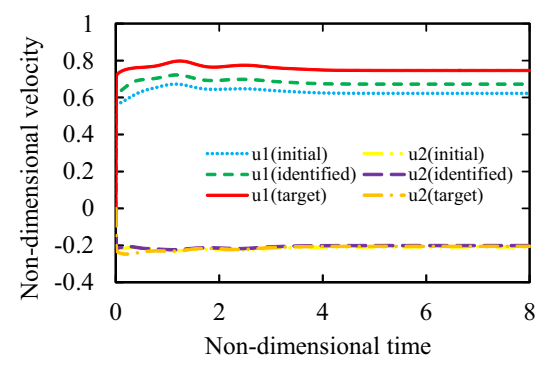

(a) In case of region $\mathrm{A}(\omega=2 \pi)$.

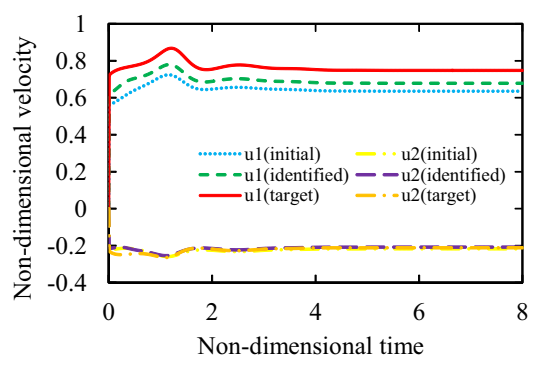

(d) In case of region $\mathrm{A}(\omega=6 \pi)$.

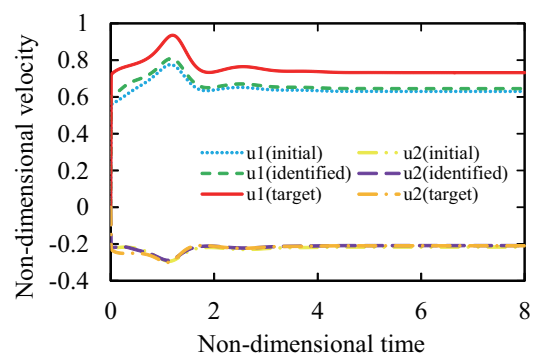

(g) In case of region $\mathrm{A}(\omega=10 \pi)$.

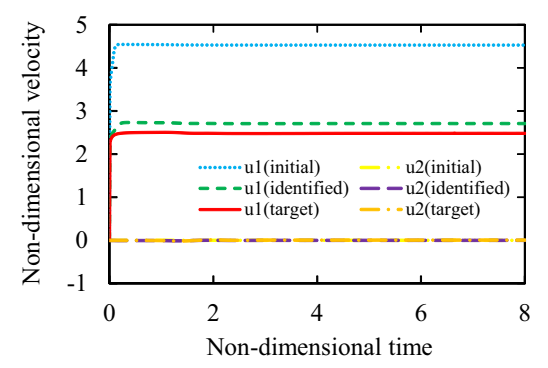

(b) In case of region $B(\omega=2 \pi)$.

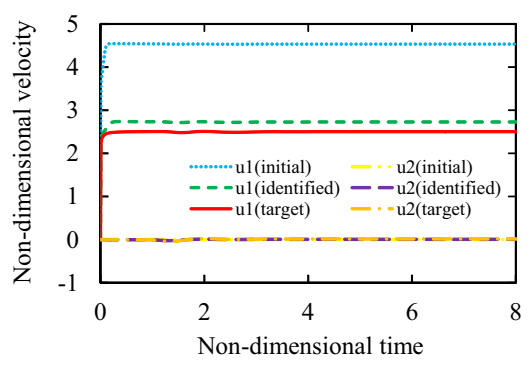

(e) In case of region $B(\omega=6 \pi)$.

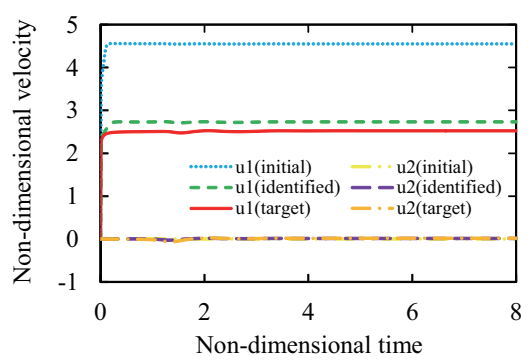

(h) In case of region $B(\omega=10 \pi)$.

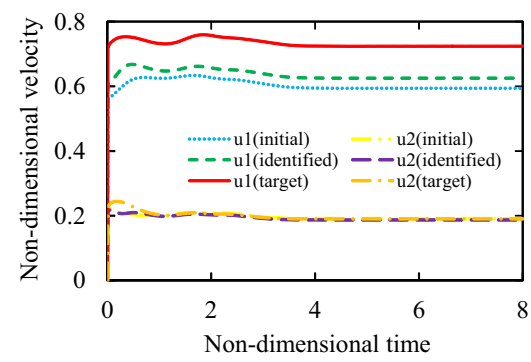

(c) In case of region $C(\omega=2 \pi)$.

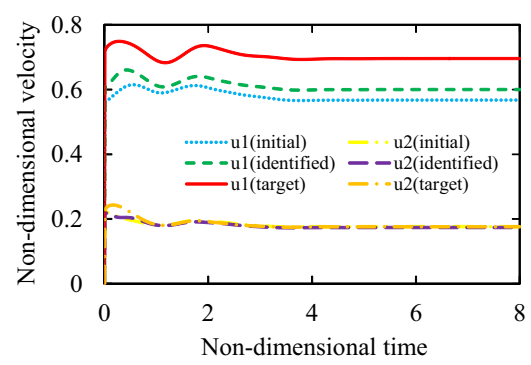

(f) In case of region $C(\omega=6 \pi)$.

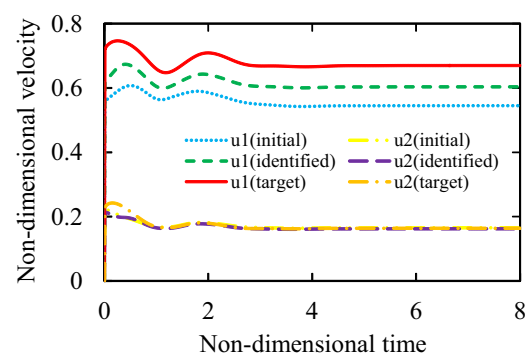

(i) In case of region $\mathrm{C}(\omega=10 \pi)$.

Fig. 11 Comparison of time history of flow velocity at the center point for $\omega=2 \pi, 6 \pi, 10 \pi$. It is seen that the non-dimensional velocity after identification is close to the target velocity in comparison with that in initial shape.

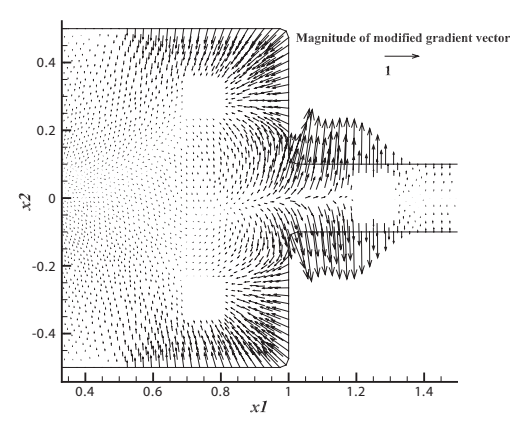

(a) In case of $\omega=2 \pi$.

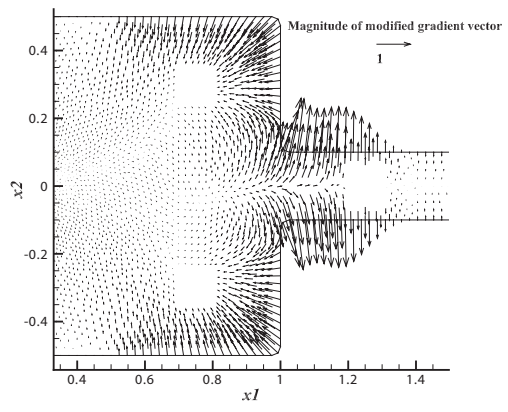

(b) In case of $\omega=6 \pi$.

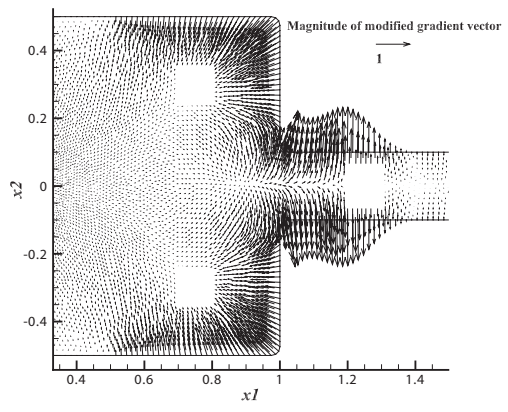

(c) In case of $\omega=10 \pi$.

Fig. 12 Distribution of the modified gradient $G_{i}^{*}$ at zero iteration. It is seen that distribution of modified gradient is almost same in all cases. 


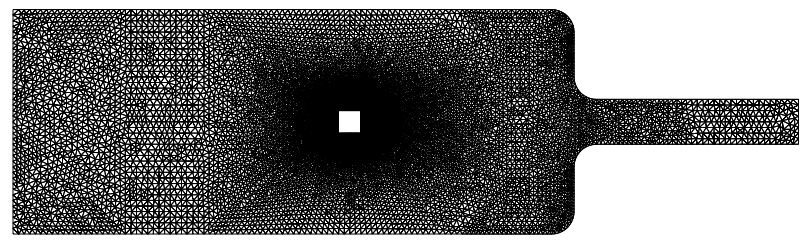

Fig. 13 Finite element mesh in case of $R=0.2$ for $\omega=2 \pi$. The vertex shape in initial mesh is changed in comparison with the previous numerical study.

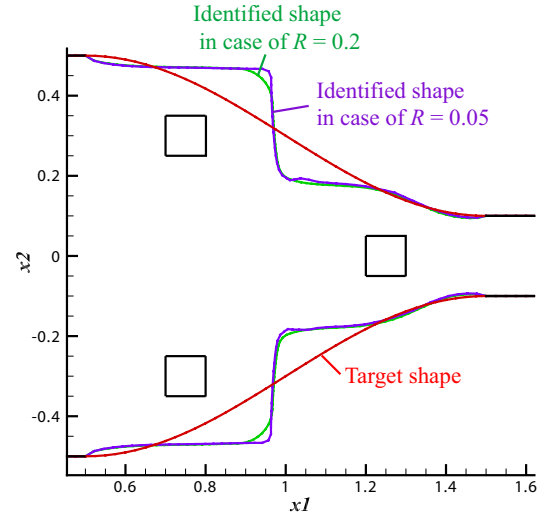

Fig. 14 Comparison of identified shape in case of $R=0.05$ and identified shape in case of $R=0.2$ for $\omega=2 \pi$. It is seen that the identified shape in case of $R=0.2$ is little bit close to the target shape in comparison with that in case of $R=0.05$, but the identified shape is almost same in case of $R=0.05$ and $R=0.2$.

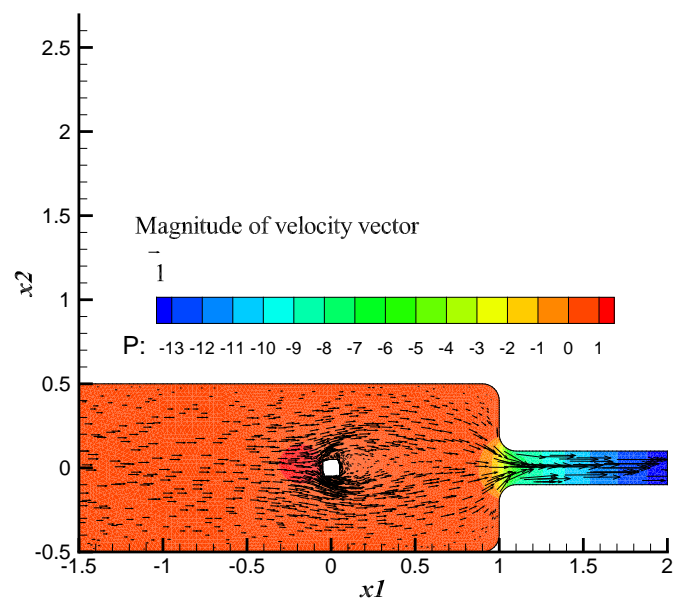

(a) Whole domain.

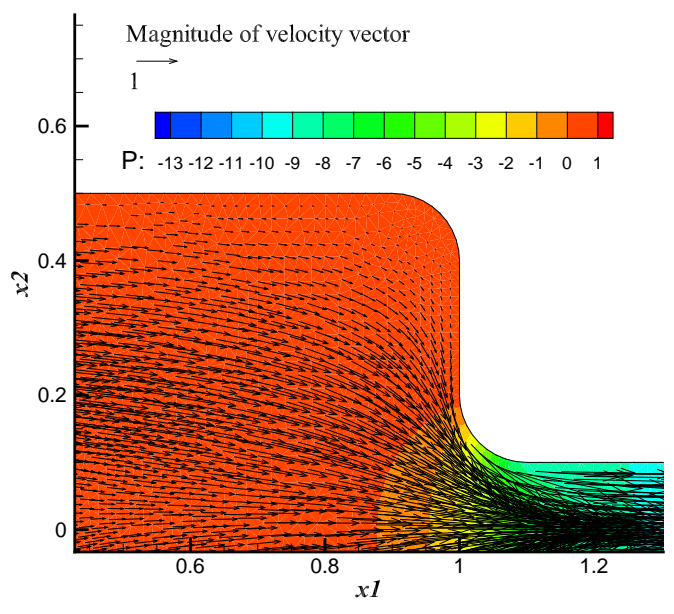

(b) Enlarged version near the vertex

Fig. 15 Distribution of velocity vector and pressure contour at $T=8.0$. It is seen that the flow field stagnant near the vertex.

From the results of numerical experiments, it can be said that the appropriate shape can be obtained such that the computed velocity is close to the target velocity. On the other hand, it is found that it is unusual for the target shape to be appropriately obtained. Looking at the results in detail, in Fig. 11, it is seen that the difference between non-dimensional velocity at the initial iteration and target velocity is large in target region B, whereas that it is small in target regions $\mathrm{A}$ and $\mathrm{C}$. It is also found that the identified shape is close to the target shape near target region $\mathrm{B}$, but distant from the target shape near target regions $\mathrm{A}$ and $\mathrm{C}$. This suggests that, the sensitivity to shape updating increases if the difference between the non-dimensional velocity at initial iteration and target velocity is high. (See Fig. 12.) Shape identification analysis using the initial shape shown in Fig. 13 was also carried out to investigate the dependence of the identified shape on the initial shape. The radius $R$ at vertex is 0.2 , and the non-dimensional angular velocity $\omega$ is given as $2 \pi$. The total number of nodes and elements are 11902 and 23246, respectively, and the time increment $\Delta t$ and time steps are set at 0.008 and 1000. A comparison of the identified shapes for $R=0.2$ and 0.05 is shown in Fig. 14. This result shows that the identified shape remains almost the same even if the initial shape is changed. The distribution of the velocity vector and the pressure contour is shown in Fig. 15. Figure 15(b) is an enlarged version of Fig. 15(a) near the vertex. From this result, it can be seen that the flow field is stagnant near the vertex. This is also a reason why the identified shape remains 
virtually unchanged. It therefore appears difficult to carry out shape updating in the convection flow field near an abruptly narrowing section.

Next, numerical test by changing the position of the rotational body is shown as follows. The non-dimensional angular velocities $\omega$ are given as $2 \pi$. The total number of nodes and elements are 10480 and 20430, and the other computational conditions are same as the above study. The computational model and the boundary conditions are shown in Fig. 16. The finite element mesh shown in Fig. 17 is employed. A comparison of the identified shapes for the present model and the model of previous example (Fig. 7(a)) is shown in Fig. 18.

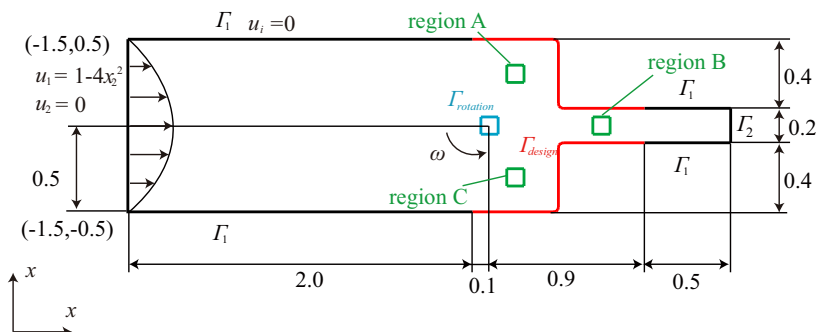

Fig. 16 Computational model, target regions and boundary condition. The position of rotational body is changed in comparison with the model in previous study, and the position of target regions is same as the model in previous study.

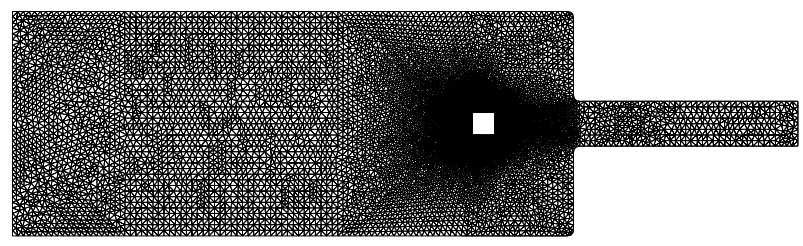

Fig. 17 Finite element mesh. The position of the rotational body is changed in comparison with the model in previous section.

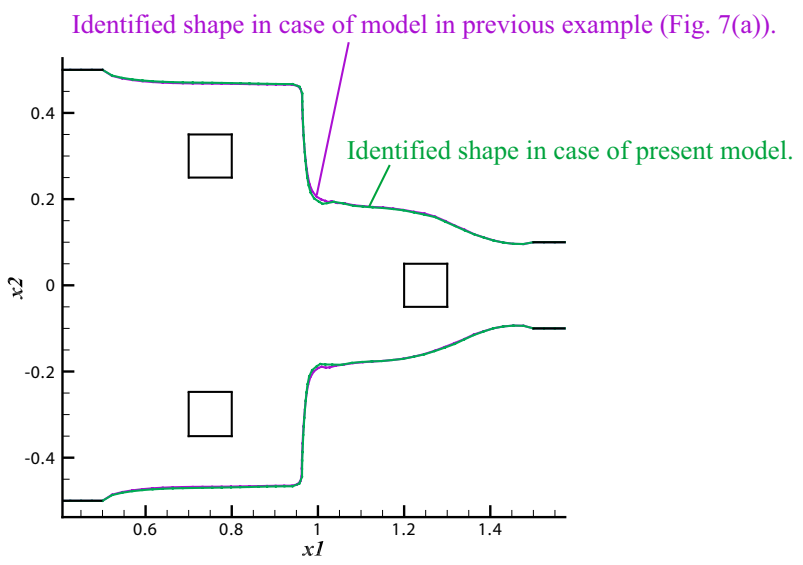

Fig. 18 Comparison of identified shape in case of present model and identified shape in case of model in previous example (Fig. 7(a)). It is seen that the identified shape in case of the present model is similar to example (Fig. 7(a)) that in case of the model in previous section.

From this result, it is found that even if the position of the rotational body is changed, the identified shape is similar to that in the previous example. Therefore, we challenged to the problem changing the size of the rotational body. However, the numerical instability occurs in the flow analysis, and the flow analysis could not be carried out. This problem is also one of future works in this study.

\section{Conclusions}

In this paper, we have presented outer shape optimization problems considering a rotational body in a flow field based on the adjoint variable and the finite element methods. The incompressible Navier-stokes equation was employed as the governing equation, and the finite element Galerkin procedure and the backward difference method, respectively was applied to discretize in space and time. The same discretization method was applied to the adjoint equation. In the numerical experiments, numerical tests were carried out for shape identification considering a rotational body. Consequently, it was seen that the drag and the lift coefficients are in good agreement with the data shown in the References (Chen et al., 1991). In addition, in the shape identification analysis, it was found that the rotational velocity does not significantly affect the identified shape. Moreover, it appears that the sensitivity of the shape update increases if there is a large difference between the stated value of the performance function at the initial iteration and the target value, and that the identified shape does not depend on the initial shape. In this study, the first order triangular element is employed for 
the variables $p, W_{p}, p^{*}$ and $W_{p}^{*}$, and the second order triangular element is used for the variables $u_{i}, W_{u_{i}}, u_{i}^{*}$ and $W_{u_{i}}^{*}$ for the interpolation of the state and the adjoint variables. If the LBB condition is satisfied, the element type can be changed, e.g., via mixed interpolation FEM using the stabilized bubble function element. It therefore is necessary to investigate the dependence of the element type on the optimal shape. This remains as a future study.

\section{Acknowledgement}

This work was supported by the Program for High Reliable Material Design and Manufacturing at Nagaoka University of Technology.

\section{References}

Azegami, H., Solution to domain optimization problems, Transactions of the Japan Society of Mechanical Engineers, Series A, Vol.60, No.574 (1994), pp.1479-1486 (in Japanese).

Azegami, H., Regularized solution to domain optimization problems, Transactions of the Japan Society for Industrial and Applied Mathematics, Vol.24, No.2 (2014), pp.83-138 (in Japanese).

Behr, M. and Tezduyar, T., The Shear-Slip Mesh Update Method, Computer Methods in Applied Mechanics and Engineering, Vol.174, (1999), pp.261-274.

Chen, Y., Ou, Y. and Pearlstein, A. J., Development of the wake behind a circular cylinder impulsively started into rotatory and rectilinear motion: intermediate rotation rates, NASA contractor report 187512, I case report No. 91-10, (1991), pp.3-62.

Hecht, F., New development in FreeFem++, Journal of Numerical Mathematics, Vol.20, No3-4, (2012), pp.251-265.

Imai, I., Fluid mechanics (First part), Shokabo Co., Ltd., (1973), pp.275-277.

Jameson, A., Aerodynamic shape optimization using the adjoint method, Lecture at the Von Karman Institute, Brussels, 2003.

Katamine, E. and Azegami, H., Solution to viscose flow field domain optimization problems (Approach by the traction method), Transactions of the Japanese Society of Mechanical Engineers, Series B, Vol.60, No.579 (1994), pp.38593866 (in Japanese).

Katamine, E. and Azegami, H., Domain optimization analysis of viscous flow field: in the case of considering convective term, Transactions of the Japanese Society of Mechanical Engineers, Series B, Vol.61, No.585 (1995a), pp.16461653 (in Japanese).

Katamine, E. and Azegami, H., Domain optimization analysis of potential flow field, Transactions of the Japanese Society of Mechanical Engineers, Series B, Vol.61, No.581 (1995b), pp.103-108 (in Japanese).

Katamine, E., Azegami, H. and Yamaguchi, S., Shape identification analyses of potential flow field: prescribed problems of pressure distribution and solution by the traction method, Transactions of the Japanese Society of Mechanical Engineers, Series B, Vol.64, No.620 (1998), pp.1063-1070 (in Japanese).

Katamine, E., Azegami, H. and Kojima, M., Boundary shape determination on steady-state heat conduction fields, Transactions of the Japanese Society of Mechanical Engineers, Series B, Vol.65, No.629 (1999), pp.275-281 (in Japanese).

Katamine, E., Azegami, H. and Matuura, Y., Solution to shape identification problem on unsteady heat-conduction fields, Transactions of the Japanese Society of Mechanical Engineers, Series B, Vol.66, No.641 (2000), pp. $227-234$ (in Japanese).

Katamine, E., Azegami, H., Tsubata, T. and Ito, S., Solution to shape optimization problems of viscose flow field, International Journal of Computational Fluid Dynamics, Vol.19, No.1 (2005), pp.45-51.

Katamine, E., Azegami, H. and Hirai, M., Solution of shape identification problems on thermoelastic solids, International Journal of Computational Methods, Vol.3, No.3 (2006), pp.279-293.

Katamine, E., Kawase, Y. and Azegami, H., Shape optimization of forced heat-convection fields, Transactions of the Japanese Society of Mechanical Engineers, Series B, Vol.73, No.733 (2007), pp.1884-1891 (in Japanese).

Katamine, E., Iwata, Y. and Azegami, H., Shape Optimization of Un-steady Heat-conduction Fields for Thermal Dissipation Maximization, Transactions of the Japanese Society of Mechanical Engineers, Series B, Vol.74, No.743 (2008a), pp.1609-1616 (in Japanese).

Katamine, E., Nishihashi N. and Azegami H., Shape optimization of steady-state viscous flow fields for drag minimization 
and lift maximization, Transactions of the Japanese Society of Mechanical Engineers, Series B, Vol.74, No.748 (2008b), pp.2426-2434 (in Japanese).

Katamine, E., Mikada, S. and Azegami, H., Shape optimization of steady-state heat-conduction fields considering temperature dependency of thermal conductivity coefficient, Transactions of the Japanese Society of Mechanical Engineers, Series B, Vol.76, No.772 (2010), pp.2188-2195 (in Japanese).

Katamine, E., Yoshioka, H., Matsuura, K. and Azegami, H., Shape optimization of thermoelastic fields for mean compliance minimization, Transactions of the Japanese Society of Mechanical Engineers, Series C, Vol.77, No.783 (2011), pp.4015-4023 (in Japanese).

Katamine, E., Kiriyama K. and Azegami, H., Multi-objective shape optimization in forced heat-convection fields, Transactions of the JSME, Vol. 79, No.806 (2013), pp.2239-2253 (in Japanese).

Katamine, E. and Imai, S., Solution to shape identification of unsteady natural convection fields to control temperature distribution, Transactions of the JSME, Vol. 82, No.833 (2016), pp.1-12 (in Japanese).

Katamine, E. and Arai, Y., Multi-objective shape optimization for maximizing stiffness in thermo-elastic fields, Transactions of the JSME, Vol. 83, No.845 (2017), pp.1-13 (in Japanese).

Kiriyama, Y., Katamine, E. and Azegami, H., Shape optimisation problem for stability of Navier-Stokes flow field, International Journal of Computational Fluid Dynamics, Vol.32, (2018), pp.68-87.

Matsumoto, J., Identification analysis for incompressible viscous flow using stabilized bubble function finite element method, Journal of applied mechanics, Vol.6, (2003), pp.267-274 (in Japanese).

Nakajima, S., and Kawahara, M., Shape optimization of a body in compressible inviscid flows, Computer Methods in Applied Mechanics and Engineering, Vol.197, (2008), pp.4521-4530.

Nakajima, S., and Kawahara, M., Two dimensional shape optimization using partial control and finite element method for compressible flows, Computer Methods in Applied Mechanics and Engineering, Vol.199, (2010a), pp.3111-3124.

Nakajima, S., and Kawahara, M., New finite element formulation based on bubble function interpolation for the transient compressible Euler equations, International Journal for Numerical Methods in Biomedical Engineering, Vol.26, No.8 (2010b), pp.1030-1049.

Ootsuka, K. and Takashi, T., Finite element analysis using mathematical programming language FreeFEM++, (2014), KYORITSU SHUPPAN CO., LTD. (In Japanese).

Pironneau, O., On optimum profiles in Stokes flow, Journal of Fluid Mechanics, Vol.59, Part1 (1973), pp.117-128.

Pironneau, O., On optimum design in fluid mechanics, Journal of Fluid Mechanics, Vol.64, Part1 (1974), pp.97-110.

Pironneau, O., On optimal shape design for elliptic systems, (1984), Springer-Verlag.

Tabata, M., Finite element analysis for fluid problems, Mathematical science, No.417, (1998), pp.13-19 (In Japanese).

Yagi, H. and Kawahara, M., Shape optimization of a body located in low Reynolds number flow, International Journal for Numerical Methods in Fluids, Vol.48, (2005), pp.819-833.

Yagi, H. and Kawahara, M., Optimal shape determination of a body located in incompressible viscous flow, Computer Methods in Applied Mechanics and Engineering, Vol.196, (2007), pp.5084-5091. 